\title{
A Message from the Editor
}

Dear Colleagues,

With this number of the journal, 30.1, it's a pleasure to unveil a new design for Renaissance and Reformation / Renaissance et Réforme. Along with the cover, the interior of the journal has also been redesigned to create a fresh look. The new design is the result of a two-year process that involved consultation with colleagues and members of the editorial board at two annual meetings of the Canadian Society for Renaissance Studies. Special thanks to our publishers, Becker Associates, for their patient and expert work on the project.

Alan Shepard, Editor

Ryerson University

Pascale Duhamel, Managing Editor

Pontifical Institute of Mediaeval Studies

\section{Un message du Directeur}

Chers collègues,

C'est avec plaisir que nous vous dévoilons, avec ce numéro 30.1, la nouvelle apparence de Renaissance and Reformation / Renaissance et Réforme. Non seulement la couverture a été entièrement transformée, mais l'intérieur a semblablement été revu dans le but de raviver l'allure de toute la revue. Ce nouveau design est l'aboutissement de deux ans de travail en collaboration avec les collègues et les membres du comité éditorial lors des deux dernières réunions annuelles de la Société Canadienne d'Études de la Renaissance. Nous remercions tout spécialement nos imprimeurs, Becker Associates, pour la patience et la compétence dont ils ont fait preuve durant ce projet.

Alan Shepard, Directeur

Ryerson University

Pascale Duhamel, Coordinatrice à l'édition

Pontifical Institute of Mediaeval Studies 